\title{
Rational Design of Photo-cleavable Alkoxyamines for Polymerization and Synthesis
}

\author{
Nicholas S. Hill ${ }^{\mathrm{a}}$ and Michelle L. Coote ${ }^{\mathrm{a} *}$
}

Theoretical calculations have been performed in order to investigate the impact of different substitution patterns on predicted photoreactivity of alkoxyamines fused to an anthraquinone chromophore. Amino and hydroxy groups (similar to those which have been previously synthesized) are introduced and their effect on excited state energies and charge transfer is assessed. Analogous to formally oxidized alkoxyamines, the charge-separated $\mathrm{n}_{\mathrm{N}} \boldsymbol{}^{*} \mathrm{state}$ can undergo mesolytic cleavage or bimolecular or $\mathrm{S}_{\mathrm{N}} 2$ reactions with nucleophiles, according to the substitution patterns and other reagents present. While homolytic cleavage is in principle promoted by triplet $\pi \pi^{*}$ states, the accessible $\pi \pi^{*}$ triplet states in this system are centered on the chromophore and unreactive. We show that the reactive $\mathrm{n}_{\mathrm{N}} \pi^{*}$ state, which bears a negative charge, is stabilized by hydroxy substitution while amino substitution will destabilize it. After mesolysis to a carbon centred radical, the nitroxide radical re-forms; however, when carbocations are produced the remaining open-shell singlet is stable and unable to undergo coupling with the carbocation.

\section{Introduction}

Nitroxide-mediated polymerization (NMP) is an important industrial process, used for controlling the molecular weight and architecture in free-radical polymerization. ${ }^{1-3}$ It works by establishing a dynamic equilibrium between the active propagating radical and a dormant alkoxyamine (Scheme 1).

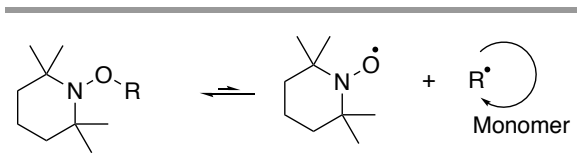

Scheme 1. Nitroxide mediated polymerization

A key drawback with nitroxide mediated polymerization, however, is the requirement for elevated temperatures can promote unwanted side reactions. ${ }^{4-13}$ As a result, the development of photochemical NMP (PNMP) is an important goal as the activation of the nitroxide species by light, rather than thermal energy, can occur at ambient reaction conditions that will reduce energy costs as well as the prevalence of side reactions. ${ }^{14-16}$

Unfortunately, the development of effective PNMP agents for a wide range of monomer units has proven difficult, with recent experimental work demonstrating that NMP-like reactivity is highly dependent on both the choice of chromophore and choice of monomer unit. ${ }^{14-20}$ Recently we studied two systems that are able to control some but not all classes of monomer tested, ${ }^{15}, 17$ and have shown that this variable behaviour may be in part due to a preference for mesolytic rather than homolytic dissociation (Scheme 2). ${ }^{21}$ Mesolytic cleavage, which occurs from the $n_{N} \pi^{*}$ rather than $\pi \pi^{*}$ or $n_{O} \pi^{*}$ excited states, results in the formation of a radical and a cation. The ultimate location of these species is dependent on the respective stabilities of these species, i.e. nitroxide radical and carbocation versus oxoammonium cation and carbon-centred radical. Only the former pathway leads to effective PNMP, the latter at best leading to initiation of cationic polymerization.

Whilst potentially detrimental to NMP reactivity, the prevalence of mesolytic cleavage does offer synthetic opportunities. Recently, experimental and theoretical work demonstrated that oxidized alkoxyamines are capable of alkylating, even methylating, nucleophiles under oxidative conditions. $^{22-25}$ These processes required formal oxidation, performed electrochemically. A simpler approach would be to use PNMP agents primed to undergo mesolytic cleavage. Here, we aim to design organic, photoactive alkoxyamines that can be tuned to enhance mesolytic cleavage, for use in photochemical alkylation reactions.

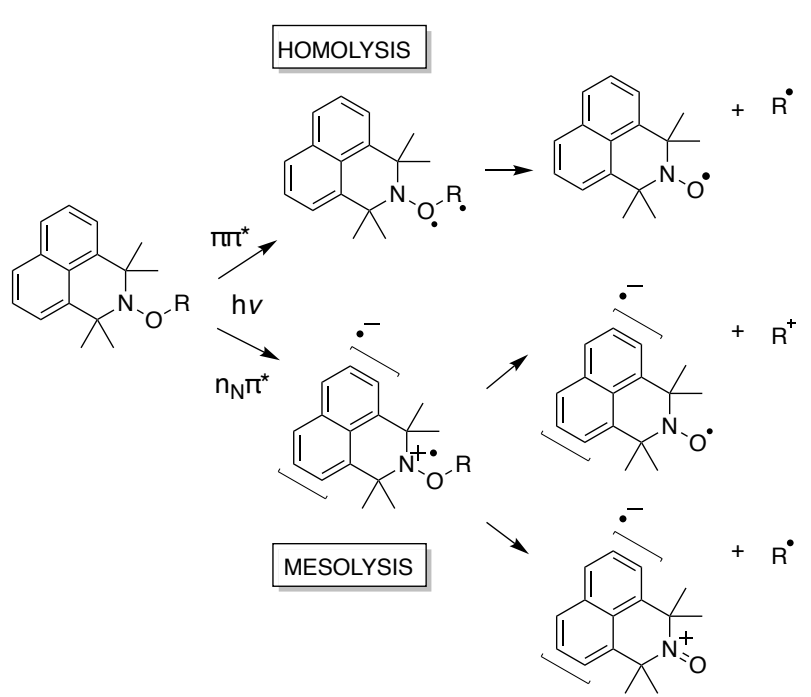

Scheme 2. Alternative homolysis and mesolysis photocleavage pathways of azaphenalenes, as studied in Ref 21 . Note that the diradical of the $\pi \pi^{*}$ is drawn across the $\mathrm{O}-\mathrm{R}$ bond to emphasize that diradicals centered on the chromophore do not undergo homolytic cleavage. 
<smiles>[R]ON1C(C)(C)c2c([X])c3c(c([X])c2C1(C)C)C(=O)c1c([X])c([X])cc([X])c1C3=O</smiles>

Anthraquinone-Alkoxyamine Scaffold<smiles>O=C1c2ccccc2C(=O)c2ccccc21</smiles>

$A Q$<smiles>[R]ON1C(C)(C)c2cc3c(cc2C1(C)C)C(=O)c1ccccc1C3=O</smiles>

$A Q-R$<smiles>[R]C(C)CCCC(=O)OCC</smiles><smiles>[R]ON1C(=O)c2cc3c(cc2C1(C)C)C(=O)c1c(N)ccc(N)c1C3=O</smiles>

1,4,5-TAAQ-R<smiles>[R]ON1C(C)(C)c2cc3c(c(N)c2C1(C)C)C(=O)c1cccc(N)c1C3=O</smiles>

1,5-DAAQ-R<smiles>[R]ON1C(C)(C)c2c(N)c3c(c(N)c2C1(C)C)C(=O)c1c(N)ccc(N)c1C3=O</smiles>

1,4,5,8-TAAQ-R<smiles>[R]ON1C(C)(C)c2cc3c(cc2C1(C)C)C(C)(C)c1cc(O)c(O)c2c1C(=O)c1c(cc(c(O)c1O)C3=O)C2(C)C</smiles>

1,2-DHAQ-R

1,2,4-THAQ-R<smiles>[R]ON1C(C)(C)c2cc3c(c(O)c2C1(C)C)C(=O)c1ccc(O)c(O)c1C3=O</smiles>

1,2,5-THAQ-R<smiles>[R]Oc1ccc2c(c1O)C(=O)c1c(O)c3c(c(O)c1C2=O)C(C)(C)N(O[R])C3(C)C</smiles>

1,2,5,8-THAQ-R

Scheme 3. Anthraquinone-Alkoxyamine substitution variations considered in this study.

\section{COMPUTATIONAL METHODOLOGY}

Electronic structure calculations were performed with the Gaussian 16.E01 software package. ${ }^{26}$ All open- and closedshell SCF calculations were performed with density functional theory (DFT), and excited state energies and electrostatic surfaces were obtained with TD-DFT. As in our previous study, ${ }^{21}$ the meta-GGA, M06-2X density functional ${ }^{27}$ was employed alongside the $6-31+G(d, p)$ basis set for all types of calculation, as this combination of functional and basis set has been shown to produce ground and excited state energies with qualitative accuracy. ${ }^{28}$ Solvent effects were obtained with the SMD universal solvent model, ${ }^{29}$ using ethyl ethanoate as the solvation environment so as to mimic a bulk acrylic monomer-like solvent. For ground state reactions, gas-phase Gibbs free energies were calculated using ideal gas partition functions and combined with solvation energies so as to obtain solution Gibbs free energies via thermocycle; for the excited states, energies in solution were obtained directly. Free energies were also obtained directly for the singlet-triplet energy gaps, to ensure the correct states were being optimised.

\section{RESULTS AND DISCUSSION}

\section{Excited States of Anthraquinone-Alkoxyamines}

As our initial test set, we considered the anthraquinonealkoxyamines in Scheme 3. These species are simple derivatives of known PNMP agents ${ }^{14-20}$ but use a chromophore that is an established Type II photoinitiator that can be tuned through ring substitution. ${ }^{30-34}$ As leaving groups we consider models of the propagating radical in styrene (STY), ethyl propanoate (EP) and ethyl isobutyrate (EIB) polymerization, as well as methyl (Me), so as to evaluate the potential for photoactivated methylation. For brevity, the different structures are named throughout to reflect the substitution of the anthraquinone moiety.

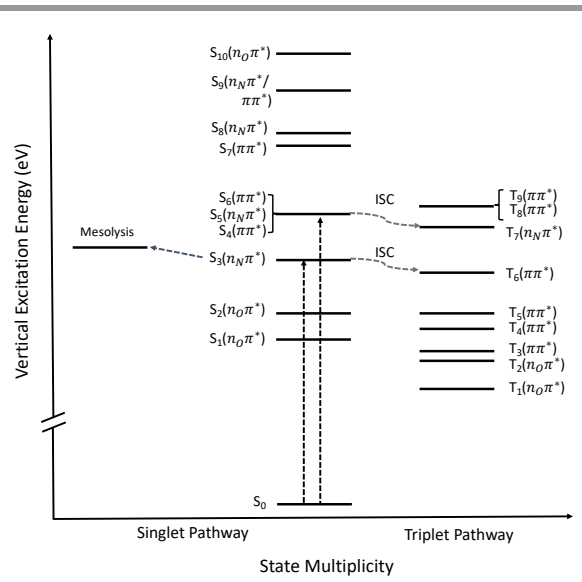

Figure 1. Simplified Jablonski diagram for AQ-Me

Figure 1 shows a simplified Jablonski diagram for AQ-Me. The extensive conjugation, keto groups, and nitrogen heteroatom present in the alkoxyamines studied give rise to $\pi \pi^{*}, n_{O} \pi^{*}$, and $n_{N} \pi^{*}$ low-lying excited states, respectively. The $\pi \pi^{*}$ and $n_{O} \pi^{*}$ states are centred on the anthraquinone moiety and are thus unreactive toward NO-R scission. TDDFT calculations show that the energies of these states are largely unaffected upon introduction of the 2,2,5,5tetramethyl-1-pyrrolidinyloxy moiety, due to the lack of conjugation between the different sources of electrons (Figure 2). Although centred on the $\mathrm{N}-\mathrm{O}$ group, the energy of the lowest $n_{N} \pi^{*}$ states, are also relatively unaffected by 
the different R-groups bonded to the alkoxyamine moiety (Figure 2).

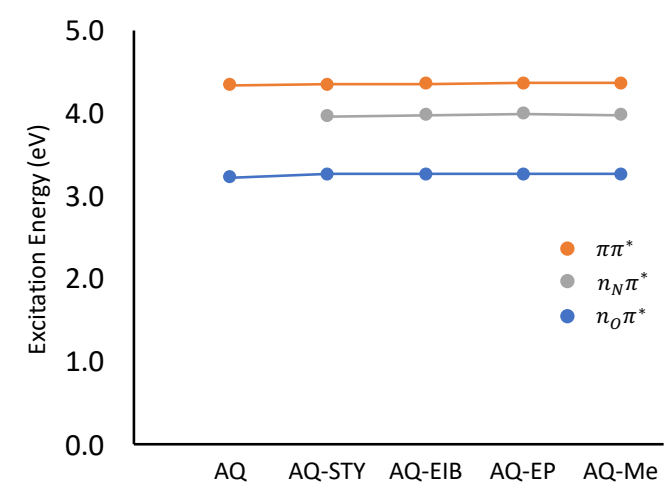

Figure 2. $n_{O} \pi^{*}, n_{N} \pi^{*}$, and $\pi \pi^{*}$ excited state energies for anthraquinone, and alkoxyamine-based derivatives.

The excited state capable of leading to mesolytic NO-R cleavage is the $n_{N} \pi^{*}$ state. As seen in Figure 1, this can occur directly from $\mathrm{S}_{3}\left(n_{N} \pi^{*}\right)$, or via intersystem crossing from $\mathrm{S}_{7}\left(\pi \pi^{*}\right)$ to $\mathrm{T}_{7}\left(n_{N} \pi^{*}\right)$. The $n_{N} \pi^{*}$ excited state results in the transfer of a non-bonding electron from the nitrogen atom into the $\pi^{*}$-system of the anthraquinone, resulting in a biradical charge-separated state. The radical cation centred on the nitrogen therefore possesses the valency necessary for mesolysis. This phenomenon is shown in Figure 3, an electrostatic potential (ESP) difference plot that shows that, upon excitation to the $n_{N} \pi^{*}$ state, there is a build-up of negative charge on the anthraquinone moiety and a concomitant build-up of positive charge around the nitrogen atom. (a)

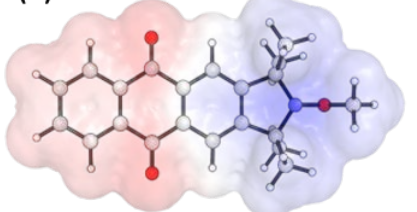

(b)

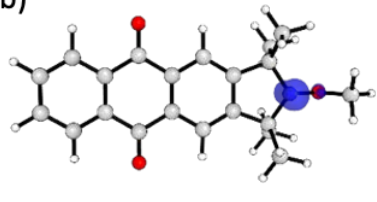

Figure 3 a) Change in electrostatic potential upon excitation to $n \pi^{*}$ excited state of methyl-anthraquinone-alkoxyamine. Blue regions correspond to a decrease in electrostatic potential, and red regions correspond to an increase in electrostatic potential, upon excitation. b) Spin density of methyl-anthraquinone-alkoxyamine radical cation.

However, compared with the equivalent, formally oxidized radical cation (Figure $3 \mathrm{~b}$ ), the positive charge shown in Figure $3 a$ is more diffuse. This raises an interesting conundrum. On the one hand, as the $n_{N} \pi^{*}$ state becomes increasingly stabilised and increasingly charge-separated, the properties around the N-O-R moiety should approach that of the formally oxidized radical cation, and mesolysis should be promoted. On the other, as seen in Figure 1, stabilization of $\mathrm{S}_{3}\left(n_{N} \pi^{*}\right)$ would in principle bring its energy closer to that of $T_{6}\left(\pi \pi^{*}\right)$, and thus would be expected to promote intersystem crossing to the triplet manifold and thus to unreactive $\pi \pi^{*}$ and $n_{O} \pi^{*}$ triplet states. A solution to this problem is to stabilize $S_{3}\left(n_{N} \pi^{*}\right)$ to such an extent that its energy drops significantly below $T_{6}\left(\pi \pi^{*}\right)$. This is the aim of the next section.

\section{Effect of Hydroxy- and Amino- Functionalisation on Anthraquinone and Anthraquinone-Alkoxyamine Properties}

There are two ways of increasing the charge-separation and stabilizing $n_{N} \pi^{*}$ : stabilizing the formation of either the radical anion or cation. Due to the limited scope for structural variation around the nitrogen, it is simpler to stabilize the anthraquinone $\pi$-system and radical anion. This stabilization will be demonstrated by a decrease in vertical excitation energy of the $n_{N} \pi^{*}$ state, an increase in the component of the excited state dipole moment along the vector that describes the change in electron density, and an increase in localization of the radical cation on the nitrogen atom. Our previous work employing anthraquinone derivatives for visible light photoinitiation highlighted the role of substitution in stabilizing key radical anion and radical cation intermediates necessary for Type II initiation. ${ }^{30-34}$ Here, similar substitution patterns are used with the anthraquinone-alkoxyamines and their subsequent effects are assessed (Figure 4).

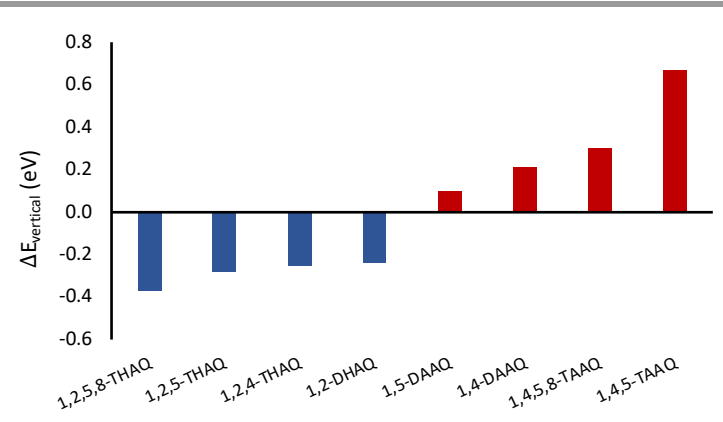

Figure 4. Average change in $n_{N} \pi^{*}$ excitation energy $(\mathrm{eV})$, relative to unfunctionalized anthraquinone-alkoxyamine, for amine (red) and hydroxyl (blue) functionalized (STY-, EP-, EIB-, Me-) anthraquinone-alkoxyamine

Figure 4 shows that amine and hydroxyl groups have opposite effects on the $n_{N} \pi^{*}$ vertical excitation energies; specifically, hydroxyl groups stabilise, and amine groups destabilise, this excited state. These effects of substitution are best demonstrated in Figure 5, which clearly shows the blue, positive charge density being "pushed" from the anthraquinone onto the alkoxyamine moiety, as the functionalization pattern moves from amine to alcohol groups. This is a clear indication that the extent of charge separation is increasing with the increase in stability of the radical anion, and the reactivity of the 1258-THAQ $n_{N} \pi^{*}$ state should resemble that of the formally oxidized species more closely than that of 1458-TAAQ or unfunctionalized $A Q$. As predicted, the observed (de)stabilization of these states is also observed in the size of the $n_{N} \pi^{*}$ state dipole (Table 1) In each case, the results of the extreme cases, 1458tetraaminoanthraquinone alkoxyamine and 1258- 
tetrahydroxyanthraquinone alkoxyamine are shown; other structures studied are shown in the SI.

(a)

(b)

(c)

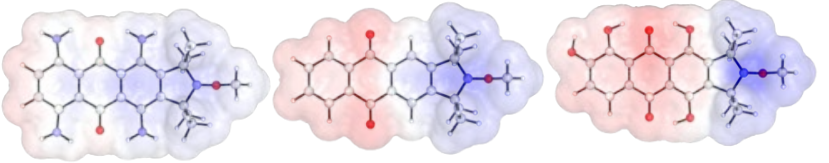

Figure 5. Change in electrostatic potential upon excitation to $n \pi^{*}$ excited state of methyl-anthraquinone-alkoxyamine derivatives with (a) amino substitution, (b) no substitution, (c) hydroxyl substitution.

Table 1. Ground and excited state dipole moment $x$-components, corresponding to the direction of charge transfer upon excitation to $n_{N} \pi^{*}$ state

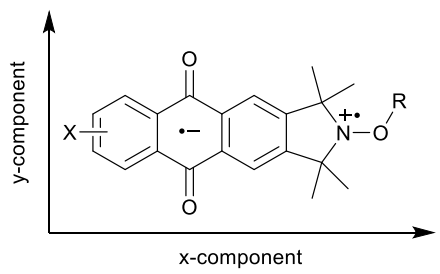

\begin{tabular}{cccc}
\hline State & $\begin{array}{c}\text { 1458-TAAQ- } \\
\text { Me }\end{array}$ & AQ-Me & $\begin{array}{c}\text { 1258-THAQ- } \\
\text { Me }\end{array}$ \\
\hline $\mathrm{S}_{0}$ & 0.4351 & 0.4797 & 0.1408 \\
$\mathrm{~S}_{\mathrm{n}}\left(n_{N} \pi^{*}\right)$ & 3.0294 & 7.6674 & 8.0705 \\
\hline
\end{tabular}

It is clear that the $\mathrm{n}_{N} \pi^{*}$ state is significantly stabilised upon hydroxy substitution due to the sigma withdrawing properties of the hydroxy groups; conversely the electron donating amino substituents destabilize it. While stabilization of $\mathrm{n}_{\mathrm{N}} \pi^{*}$ makes it more accessible and more reactive to mesolysis, it is important to consider competing intersystem crossing to the triplet manifold. To this end, simplified Jablonski diagrams for the two extreme cases, 1458-tetraaminoanthraquinone alkoxyamine and 1258tetrahydroxyanthraquinone alkoxyamine are shown in Figure 6 . In both cases the orbitals are re-ordered compared with the unsubstituted anthraquinone alkoxyamine with the net effect that the ${ }^{1} n_{N} \pi^{*}-{ }^{3} \pi \pi^{*}$ increases. This increase will limit ISC and promote reactivity on the singlet surface, which is then favoured by hydroxy substitution.

So far, attention has been drawn to the impact of functionalization on the $n_{N} \pi *$ excited state energy, and below the potential reactivity of these states is explored and discussed. A key issue one may expect from any reactivity stemming from population of the $n_{N} \pi^{*}$ is the population of the state itself. As shown in Table S2, and as expected, the oscillator intensities of the symmetry-forbidden ${ }^{1} n_{N} \pi$ * transitions are significantly smaller than that of the ${ }^{1} \pi \pi^{*}$, symmetry-allowed transitions. Importantly for $\mathrm{n}_{\mathrm{N}} \pi^{*}$ reactivity, however, the "forbidden" transition is weakly allowed (oscillator intensities are non-zero), and therefore direct population of $n_{N} \pi^{*}$ states, whilst spectroscopically dark compared to $\pi \pi^{*}$ states, will take place to a measurable and useful extent. This is supported experimentally in the work by Barner-Kowollik et al., ${ }^{35}$ which showed how direct population of "dark" $\mathrm{n}_{N} \pi^{*}$, achieved with a tuneable laser, led to an increase in per-photon reaction efficiency.

Fluorescence and phosphorescence from singlet and triplet excited states may also lead to a decrease in, or may even prevent, excited state reactivity. It is fair to say, however, that fluorescence ought not to be an issue from a ${ }^{1} n_{N} \pi$ * state, as the inefficient coupling (from low oscillator intensity) to the ground state that prevents its efficient population will also prevent efficient fluorescence. Phosphorescence is more difficult to predict, as from Figure 6 there are multiple low-lying triplet excited states in these functionalized systems. In the case of 1258-THAQ, the system in which we expect the properties of the ${ }^{1} n_{N} \pi *$ excited state to be promoted, the closest triplet state is $T_{6}\left(n_{N} \pi^{*}\right)$. From El-Sayed, we expect intersystem crossing to this state to be slow.
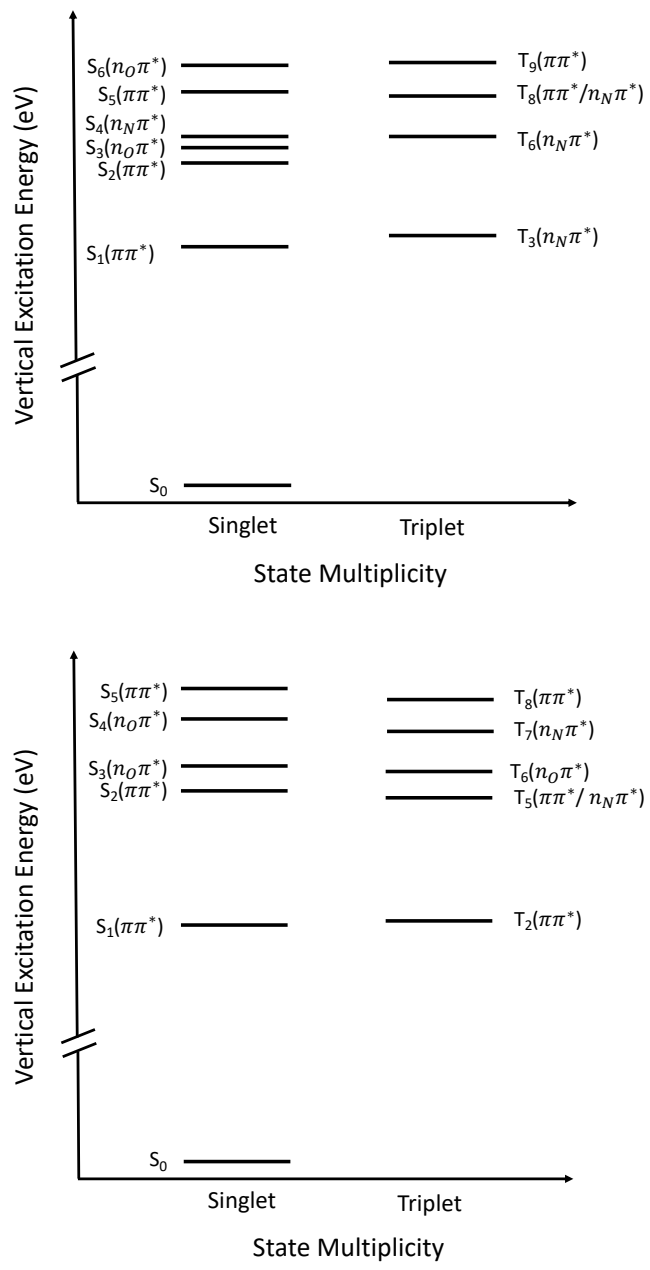

Figure 6. Simplified Jablonski diagrams for 1258-THAQ-Me (top) and 1458-TAAQ-Me (bottom). For 1258-THAQ, the lowest $\mathrm{S}\left(\mathrm{n}_{N} \pi^{*}\right)$ state and nearest triplet state $\mathrm{T} 6\left(\mathrm{n}_{\mathrm{O}} \pi^{*}\right)$ have the same orbital symmetry and intersystem crossing would be expected to be slow based on El-Sayed rules. For 1458-TAAQ-Me there is no lowlying $S\left(n_{N} \pi^{*}\right)$ state and this molecule would be predicted to be unsuitable for mesolytic reactivity. 

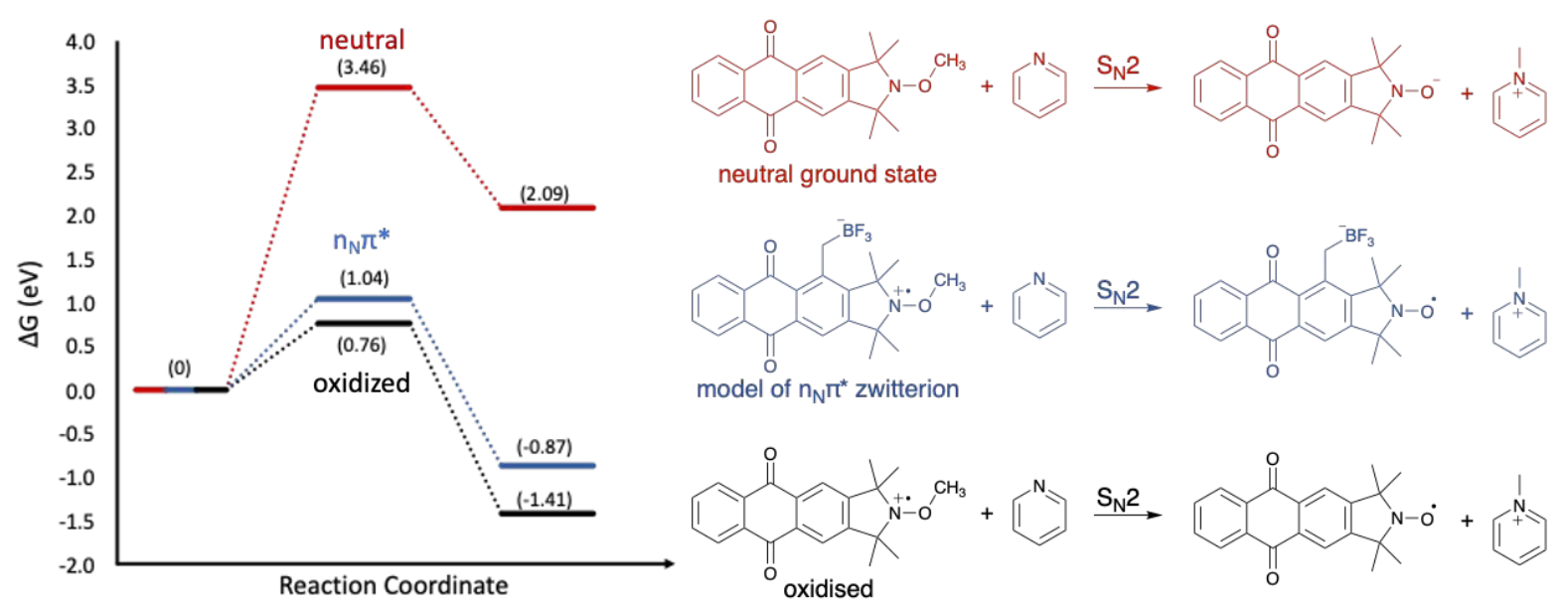

Figure 7. $\mathrm{S}_{\mathrm{N}} 2$ mechanism and relative free energies for ground state (red), oxidized (black), and zwitterionic (blue) anthraquinone-alkoxyamine.

\section{Promotion of Alkylation.}

Recently we showed that oxidized TEMPO-methyl is a potent in situ methylating agent in the presence of nucleophiles. ${ }^{25}$ Quantum chemistry calculations and isotopic labelling suggest an $\mathrm{S}_{\mathrm{N}} 2$ mechanism, resulting in the transfer of a methyl cation to form the methylated nucleophile and the corresponding nitroxide radical. The activation barrier of the $\mathrm{S}_{\mathrm{N}} 2$ reaction is shown to be significantly decreased upon oxidation, compared with the same reaction on the ground state singlet surface. Closed and open-shell calculations on the ground state and radical cation anthraquinone alkoxyamines, respectively, demonstrate that similar catalysis of the $\mathrm{S}_{\mathrm{N}} 2$ methylation mechanism can be expected upon formal oxidation (Figure 7). In reality, the $n_{N} \pi^{*}$ excited state exists as a zwitterionic biradical species, however accurately modelling this species is difficult with standard TD-DFT methods. It is well established that TD-DFT fails with charge-transfer excited states, and optimization of transition states of excited states, especially those higher into the excited state manifold. ${ }^{36}$ Instead, the reactivity of the $n_{N} \pi^{*}$ state is explored here as a ground state zwitterionic species. To this end, a negatively charged boron trifluoride $\left(\mathrm{BF}_{3}\right)$ functional group is attached to the anthraquinone moiety close to the nitroxide moiety, resulting in a through-space electrostatic stabilization effect of the localized radical cation. The relative energies for the zwitterionic reaction are also shown in Figure 7. Due to the stabilization of the anthraquinone-alkoxyamine-methyl radical cation the activation energy of the $\mathrm{S}_{\mathrm{N}} 2$ reaction increases slightly, however the calculations suggest that the positivenegative electrostatic interaction would not drastically alter the photoreactivity around the alkoxyamine.

\section{On the Fate of the Anthraquinone-centred Radical Anion}

So far, calculations and discussion have focussed on the formation and reactivity of the charge-separated $n_{N} \pi^{*}$ excited state. If upon mesolysis the anthraquinone derivatives are to be chemically useful, for example in PNMP, the relaxation of the anthraquinone-centred radical anion, in combination with either the oxoammonium or nitroxide radical, is important to consider. Figure 8 illustrates the immediate products formed upon mesolysis from the $n_{N} \pi^{*}$ state, by removal of either a cation or radical, and the Aufbau resonance structures that minimize the number of unpaired electrons.

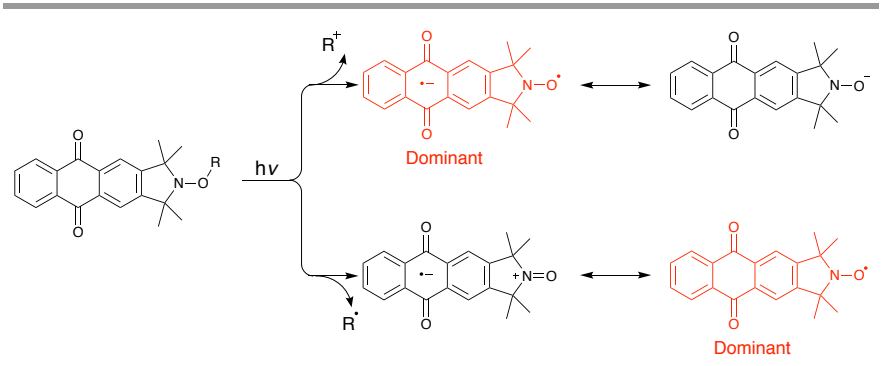

Figure 8. Formal products of photo-mesolysis and isoelectronic resonance structures.

The cleavage pathway to form the carbocation proved the simplest to study. The oxygen-centred anion (black, top structure, Figure 8), is a singlet; the biradical anion can either be a triplet or an open-shell singlet. Optimization of the biradical triplet state can be used for a subsequent broken-shell DFT calculation, which uses the optimized triplet orbitals as an SCF guess for a singlet calculation. This approach yielded the open-shell, biradical singlet species. In each case the open-shell singlet biradical is more stable than the closed-shell oxygencentred anion, sitting $70.2,93.6$ and $134.9 \mathrm{~kJ} \mathrm{~mol}^{-1}$ below the closed shell species for 1,4,5-TAAQ, AQ and 1,2,5,8-THAQ respectively. The consequence of the stability of the singlet biradical is the lack of ionic recombination between the carbocation and oxygen centred anion, as the nitroxide radical will persist. The radical anion, however, could be oxidised by coinitiators as has been reported previously. ${ }^{30-34}$

For the radical zwitterion, broken-shell DFT calculations are more difficult as both resonance structures exhibit the same charge and spin multiplicity. An alternative approach to finding a non-Aufbau SCF solution is to use the Intermediate Neglect of Differential Overlap (INDO) occupancy guess, which unfortunately simply results in convergence to the nitroxide radical SCF solution for each of the three species, suggesting 
that there is no low-lying zwitterionic solution present (black, bottom structure, Figure 8 ). Time-dependent approaches are also not viable due to severe spin-contamination issues when performed on open-shell species. However, given the apparent stability of the nitroxide radical this seems the most likely product. Relaxation upon mesolysis to this species would allow for continued controlled radical polymerization. Alternatively, in an alkylation reaction the nitroxide would be inert.

\section{CONCLUSIONS}

To conclude, a range of anthraquinone-functionalized alkoxyamines have been studied using DFT and TD-DFT, and predictions have been made about their respective (photo)reactivities. With good leaving groups, these reagents undergo mesolytic cleavage which is favoured by hydroxylfunctionalization. The products of mesolytic cleavage are either carbon-centred radicals or carbocations, with the only the former favouring nitroxide-mediated polymerization. Based on our experimental studies of oxidative cleavage, radical formation is expected to occur for (meth)acrylate polymerizations, while for styrene polymerization cationic products may be implicated. More importantly, the methyl alkoxyamines are predicted to be excellent candidates for photochemical methylation, analogous to our recently published electrochemical procedure. ${ }^{25}$ Synthetic work in this direction is currently underway.

\section{Conflicts of interest}

There are no conflicts to declare.

\section{Acknowledgements}

The authors acknowledge financial support from the Australian Research Council (ARC) Centre of Excellence for Electromaterials Science, an ARC Laureate Fellowship (to M.L.C.), generous supercomputing time from the National Computational Infrastructure, and helpful discussions with Yohann Guillaneuf, Didier Gigmes, and Jason Morris.

\section{Notes and references}

1. D. Bertin, D. Gigmes, S. R. A. Marque and P. Tordo, Chem. Soc. Rev., 2011, 40, 2189-2198.

2. R. B. Grubbs, Polym. Rev., 2011, 51, 104-137.

3. C. J. Hawker, A. W. Bosman and E. Harth, Chem. Rev., 2001, 101, 3661-3688.

4. C. Dire, J. Belleney, J. Nicolas, D. Bertin, S. Magnet and B. Charleux, J. Polym. Sci. A Polym. Chem., 2008, 46, 63336345.

5. M. Souaille and H. Fischer, Macromolecules, 2001, 34, 2830-2838.

6. A. Goto and T. Fukuda, Macromolecules, 1999, 32, 618 623.

$7 . \quad$ C. Burguière, M.-A. Dourges, B. Charleux and J.-P. Vairon, Macromolecules, 1999, 32, 3883-3890.
D. Benoit, V. Chaplinski, R. Braslau and C. J. Hawker, J. Am. Chem. Soc., 1999, 121, 3904-3920.

9. G. S. Ananchenko and H. Fischer, J. Polym. Sci. A Polym. Chem., 2001, 39, 3604-3621.

10. Y. Guillaneuf, D. Gigmes, S. R. A. Marque, P. Tordo and D. Bertin, Macromol. Chem. Phys., 2006, 207, 1278-1288.

11. M. Edeleva, S. R. A. Marque, K. Kabytaev, Y. Guillaneuf, D. Gigmes and E. Bagryanskaya, J. Polym. Sci. A Polym. Chem., 2013, 51, 1323-1336.

12. G. S. Ananchenko, M. Souaille, H. Fischer, C. Le Mercier and P. Tordo, J. Polym. Sci. A Polym. Chem., 2002, 40, 32643283.

13. G. Gryn'ova, C. Y. Lin and M. L. Coote, Polym. Chem., 2013, 4, 3744-3754.

14. M. Chen, M. Zhong and J. A. Johnson, Chem. Rev., 2016, 116, 10167-10211.

15. Y. Guillaneuf, D. Bertin, D. Gigmes, D.-L. Versace, J. Lalevée and J.-P. Fouassier, Macromolecules, 2010, 43, 2204-2212.

16. J. Morris, S. Telitel, K. E. Fairfull-Smith, S. E. Bottle, J. Lalevée, J.-L. Clément, Y. Guillaneuf and D. Gigmes, Polym. Chem., 2015, 6, 754-763.

17. S. E. Bottle, J.-L. Clement, M. Fleige, E. M. Simpson, Y. Guillaneuf, K. E. Fairfull-Smith, D. Gigmes and J. P. Blinco, RSC Adv., 2016, 6, 80328-80333.

18. Y. Guillaneuf, D.-L. Versace, D. Bertin, J. Lalevée, D. Gigmes and J.-P. Fouassier, Macromol. Rapid Commun., 2010, 31, 1909-1913.

19. M. Herder and J.-M. Lehn, J. Am. Chem. Soc., 2018, 140, 7647-7657.

20. M. Huix-Rotllant and N. Ferre, J. Phys. Chem. A, 2014, 118, 4464-4470.

21. N. S. Hill, M. J. Fule, J. Morris, J.-L. Clément, Y. Guillaneuf, D. Gigmes and M. L. Coote, Macromolecules, 2020, 53, 1567-1572.

22. L. Zhang, E. Laborda, N. Darwish, B. B. Noble, J. H. Tyrell, S. Pluczyk, A. P. Le Brun, G. G. Wallace, J. Gonzalez, M. L. Coote and S. Ciampi, J. Am. Chem. Soc., 2018, 140, 766-774.

23. C. L. Hammill, B. B. Noble, P. L. Norcott, S. Ciampi and M. L. Coote, J. Phys. Chem. C, 2019, 123, 5273-5281.

24 B. B. Noble, P. L. Norcott, C. L. Hammill, S. Ciampi and M. L. Coote, J. Phys. Chem. C, 2019, 123, 10300-10305.

25. P. L. Norcott, C. L. Hammill, B. B. Noble, J. C. Robertson, A. Olding, A. C. Bissember and M. L. Coote, J. Am. Chem. Soc., 2019, 141, 15450-15455.

26. M. J. Frisch, G. W. Trucks, H. B. Schlegel, G. E. Scuseria, M. A. Robb, J. R. Cheeseman, G. Scalmani, V. Barone, G. A. Petersson, H. Nakatsuji, X. Li, M. Caricato, A. Marenich, J. Bloino, B. G. Janesko, R. Gomperts, B. Mennucci, H. P. Hratchian, J. V. Ortiz, A. F. Izmaylov, J. L. Sonnenberg, D. Williams-Young, F. Ding, F. Lipparini, F. Egidi, J. Goings, B. Peng, A. Petrone, T. Henderson, D. Ranasinghe, V. G. Zakrzewski, J. Gao, N. Rega, G. Zheng, W. Liang, M. Hada, M. Ehara, K. Toyota, R. Fukuda, J. Hasegawa, M. Ishida, T. Nakajima, Y. Honda, O. Kitao, H. Nakai, T. Vreven, K. Throssell, J. A. Montgomery Jr., J. E. Peralta, F. Ogliaro, M. Bearpark, J. J. Heyd, E. Brothers, K. N. Kudin, V. N. Staroverov, T. Keith, R. Kobayashi, J. Normand, K. Raghavachari, A. Rendell, J. C. Burant, S. S. Iyengar, J. Tomasi, M. Cossi, J. M. Millam, M. Klene, C. Adamo, R. Cammi, J. W. Ochterski, R. L. Martin, K. Morokuma, O. Farkas, J. B. Foresman and D. J. Fox, Gaussian 09, Revision E.01, Gaussian, Inc., Wallingford CT, 2016. 
27. Y. Zhao and D. G. Truhlar, Theor. Chem. Acc., 2008, 120, 215-241.

28. A. D. Laurent and D. Jacquemin, Int. J. Quantum Chem., 2013, 113, 2019-2039.

29. A. V. Marenich, C. J. Cramer and D. G. Truhlar, J. Phys. Chem. B, 2009, 113, 6378-6396.

30. G. N. Wang, N. S. Hill, D. Zhu, P. Xiao, M. L. Coote and M. H. Stenzel, Acs Appl. Polym. Mat., 2019, 1, 1129-1135.

31. J. Zhang, N. Hill, J. Lalevee, J. P. Fouassier, J. C. Zhao, B. Graff, T. W. Schmidt, S. H. Kable, M. H. Stenzel, M. L. Coote and P. Xiao, Macromol. Rapid Commun., 2018, 39, 6.

32. J. Zhang, J. Lalevee, N. S. Hill, K. Launay, F. Morlet-Savary, B. Graff, M. H. Stenzel, M. L. Coote and P. Xiao, Macromolecules, 2018, 51, 8165-8173.

33. J. Zhang, J. Lalevee, N. S. Hill, X. T. Peng, D. Zhu, J. Kiehl, F. Morlet-Savary, M. H. Stenzel, M. L. Coote and P. Xiao, Macromol. Rapid Commun., 2019, 40, 5.

34. J. Zhang, K. Launay, N. S. Hill, D. Zhu, N. Cox, J. Langley, J. Lalevee, M. H. Stenzel, M. L. Coote and P. Xiao, Macromolecules, 2018, 51, 10104-10112.

35. J. P. Menzel, B. B. Noble, A. Lauer, M. L. Coote, J. P. Blinco and C. Barner-Kowollik, J. Am. Chem. Soc., 2017, 139, 15812-15820.

36. R. Guareschi and C. Filippi, J. Chem. Theory Comput., 2013, 9, 5513-5525. 